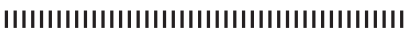

Society Awards 2016

IIIIIIIIIIIIIIIIIIIIIIIIIIIIIIIIIIIIIIIIIII

(on high prospectiveness)

\title{
Studies on the mode of action of neurotoxic insecticides
}

\author{
Koichi Hirata* \\ Odawara Research Center, Nippon-soda Co., Ltd., \\ Takada, Odawara, Kanagawa 250-0216, Japan
}

(Accepted June 6, 2016)

\begin{abstract}
Many of the commercially available insecticides target the nervous system of insects, which are important targets of insecticides. We investigated the mechanism of action of the compound acts on voltage-sensitive sodium channel (Vssc) and nicotinic acetylcholine receptor (nAChR), and the associated mechanism of resistance, mainly using an electrophysiological method. The mechanism of resistance to pyrethroids in A. aegypti, that to neonicotinoids in A. gossypii, and the mechanism of action of the natural product asperparaline A were clarified. We also demonstrated that similar compounds that interacted on the same target with slightly different substituents exhibited different effects on the target molecule with different amino acid residues at the activity site, and that natural products exhibited specific activity and selectivity to insect ion channels. (c) Pesticide Science Society of Japan

Keywords: mode of action of insecticides, insecticide resistance, neonicotinoids, pyrethroids, asperparaline, ion channels.
\end{abstract}

\section{Introduction}

Many of the commercially available insecticides, such as pyrethroids, neonicotinoids, and some other natural products, target the nervous system of insects and affect the voltage-sensitive sodium channel (Vssc) and nicotinic acetylcholine receptor (nAChR), which are transmembrane proteins that play an important role in signal transduction in the nervous system, and are important targets of insecticides.

The Vssc is composed of $\alpha$ - and $\beta$-subunits; the $\alpha$-subunit is composed of four domains, each containing six transmembrane segments (S1-S6). The $\alpha$-subunit is the main functional subunit, while the $\beta$-subunit supports it. ${ }^{1)}$ The Vssc opens due to an increase or decrease in the membrane potential, which causes an influx of sodium ions into the cell and subsequent depolarization. The Vssc is affected by various compounds, and is a target of pyrethroids, as well as tetrodotoxin, which is also known as fugutoxin. These compounds are classified according to the site of interaction; ten sites of interaction have been identified to date, and the pyrethroids are known to act on site $7{ }^{2)}$ Pyrethroids exhibit insecticidal activity by causing a delay in inactivation of the Vssc and excessively exciting the nervous system. ${ }^{3)}$

$\mathrm{nAChR}$, inhibitory-aminobutyric acid receptor, inhibitory glutamic acid receptor, and glycine receptor belong to the cys-

\footnotetext{
* To whom correspondence should be addressed.

E-mail: k.hirata@nippon-soda.co.jp

Published online August 2, 2016

(C) Pesticide Science Society of Japan
}

loop ligand-gated ion channel superfamily. ${ }^{4)} \mathrm{nAChR}$ is composed of five subunits having four-transmembrane structures to form homomers or heteromers ${ }^{5}$; upon binding with its ligand acetylcholine $(\mathrm{ACh})$, this channel opens and allows cations to penetrate. Natural products such as nicotine or neonicotinoids act on $\mathrm{nAChR}$, bind to it, and exhibit an agonistic effect. ${ }^{6-9)}$

A range of methods are available for studying the mechanism of action of compounds having insecticidal activity, including molecular biology and biochemical approaches, binding assays, optical methods using fluorescent dye, and electrophysiological methods. Among these, electrophysiological methods are the most suitable for detailed testing of the effects of actions or mechanisms of action of receptors or ion channels because such approaches identify the effects of the compound by detecting changes in current. There are three different types of electrophysiological method. One is the two-electrode voltage-clamp method (TEVC), which uses Xenopus oocytes. This method is suitable for the expression and analysis of receptors of a wide range of biological strains, especially for the analysis of variants. ${ }^{10)}$ Another method is the patch clamp method; this enables various measurements, is particularly applicable to the nerve cells of insects, and enables evaluation of the effects or activities of a compound on the expression of receptors in an insect's body. The third method is the automatic patch clamp method. This was developed to improve the low throughput of electrophysiological methods, which is one of their drawbacks, is labor-saving, and can be used to analyze a large number of compounds, while utilizing the benefits of electrophysiological features. 


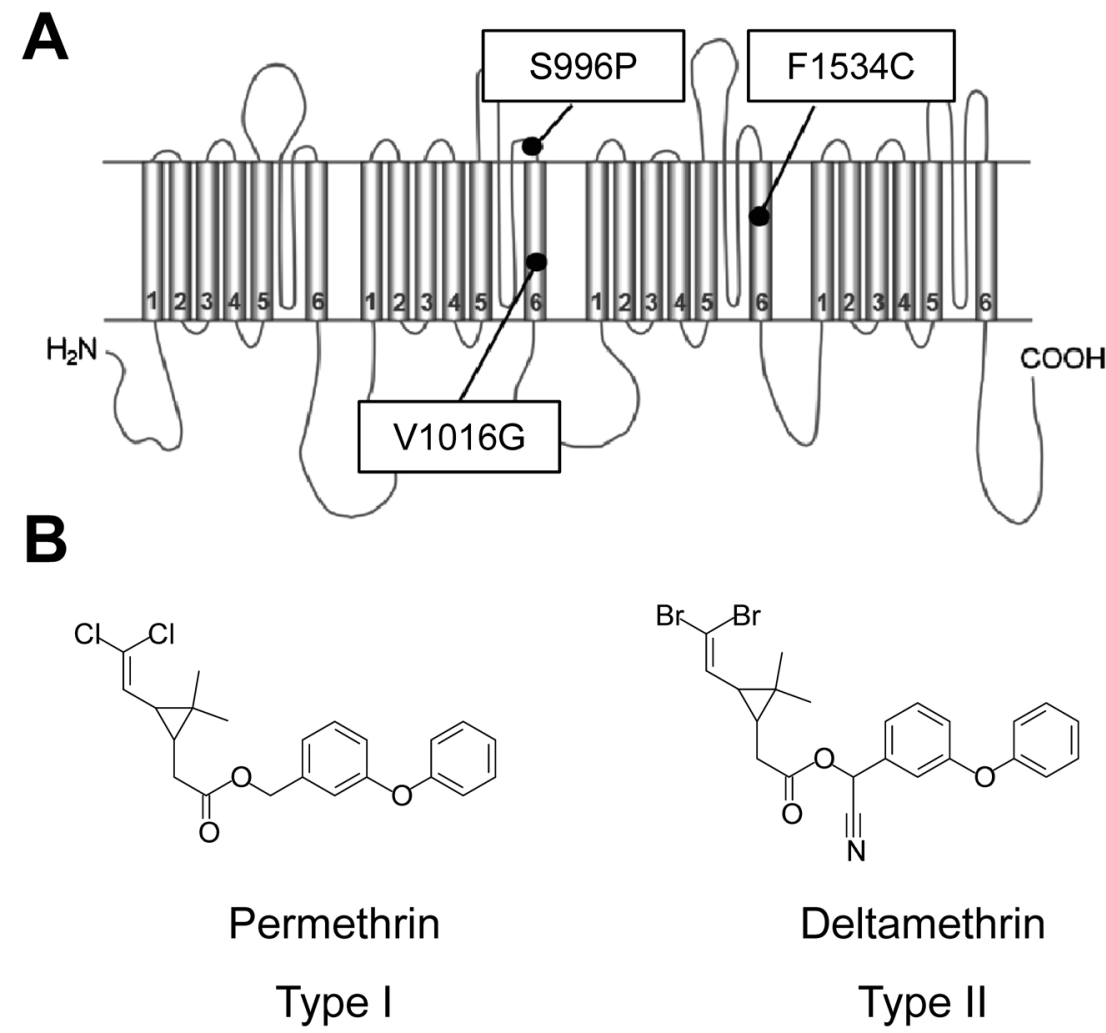

Fig. 1. Voltage-gated sodium channel and chemical structures of pyrethroid insecticides. (A) Voltage-gated sodium channel mutations of Aedes aegypti investigated in this study. (B) Chemical structures of type I (permethrin) and type II pyrethroids (deltamethrin).

Table 1. Voltage-dependence of activation of Aedes aegypti sodium channels and sensitivity of mutant channels to pyrethroids

\begin{tabular}{|c|c|c|c|c|c|c|c|c|c|c|}
\hline \multirow{3}{*}{ Vssc type } & \multirow{3}{*}{ Mutations } & \multirow{2}{*}{\multicolumn{3}{|c|}{$\frac{\text { Voltage dependence }}{\text { Activation }}$}} & \multicolumn{6}{|c|}{ Pyrethroid sensitivity } \\
\hline & & & & & \multicolumn{3}{|c|}{ Permethrin } & \multicolumn{3}{|c|}{ Deltamethrin } \\
\hline & & $n$ & $V_{1 / 2}(\mathrm{mV})$ & $k(\mathrm{mV})$ & $n$ & $\mathrm{EC}_{25}(\mu \mathrm{M})$ & Ratio & $n$ & $\mathrm{EC}_{25}(\mu \mathrm{M})$ & Ratio \\
\hline $\mathrm{AaNa}_{\mathrm{v}} \mathrm{S} 2$ & Wild-type & 8 & $-31.1 \pm 0.4$ & $3.9 \pm 0.3$ & 4 & 0.002 & - & 4 & 0.01 & - \\
\hline $\mathrm{AaNa}_{\mathrm{v}} \mathrm{R} 6$ & V1016G & 7 & $-27.5 \pm 0.7^{*}$ & $4.2 \pm 0.5$ & 5 & 0.2 & 100 & 8 & 0.02 & 2 \\
\hline $\mathrm{AaNa}_{\mathrm{v}} \mathrm{R} 7$ & F1534C & 7 & $-28.7 \pm 0.3^{*}$ & $4.0 \pm 0.2$ & 4 & 0.05 & 25 & 4 & 0.01 & 1 \\
\hline $\mathrm{AaNa}_{\mathrm{v}} \mathrm{R} 8$ & S989P & 7 & $-28.4 \pm 0.4^{*}$ & $4.3 \pm 0.3$ & 4 & 0.002 & 1 & 4 & 0.01 & 1 \\
\hline $\mathrm{AaNa}_{\mathrm{v}} \mathrm{R} 9$ & S989P+V1016G & 8 & $-32.0 \pm 0.4$ & $3.5 \pm 0.4$ & 4 & 0.2 & 100 & 4 & 0.1 & 10 \\
\hline $\mathrm{AaNa}_{\mathrm{v}} \mathrm{R} 10$ & S989P+V1016G+F1534C & 9 & $-29.4 \pm 0.8$ & $5.0 \pm 0.7$ & 4 & 2.2 & 1100 & 4 & 0.9 & 90 \\
\hline
\end{tabular}

Data were fitted with the Boltzmann equation to determine $V_{1 / 2}$, the voltage of half-maximal conductance, and $k$ the slope factor. Values represent the mean \pm standard errors. Voltage dependences were compared statistically between wild-type and mutant channels using one-way ANOVA followed by Dunnett's multiple comparison test. ${ }^{*} p<0.05$. Ratios were calculated by $\mathrm{EC}_{25}$ (mutant)/ $\mathrm{EC}_{25}$ (wild-type).

The development of resistance to insecticides is one of the most important problems encountered during insect pest management. Detoxification by P450 for example, mutation of an amino acid residue at the site of activity, and a decrease in the permeability of an insecticide due to an increased cuticle surface area are known to be involved in such resistance. ${ }^{11-13)}$ We believe that the findings of studies on the mechanisms of action of insecticides and resistance to them will contribute to the research and development of new insecticides, including ones effective against insecticide-resistant insects, and to the establishment of appropriate and effective measures for avoiding resistance.
Therefore, in the present study, we investigated the mechanism of action of the compound that acts on Vssc and nAChR, and the associated mechanism of resistance, mainly using an electrophysiological method.

\section{Voltage-sensitive sodium channel and pyrethroid resistance of Aedes aegypti}

Aedes aegypti is the major mosquito vector of dengue fever, yellow fever, and chikungunya fever. Dengue fever causes more illness and death in humans than any other arboviral disease. Mosquito control is mainly achieved by pyrethroids because of 

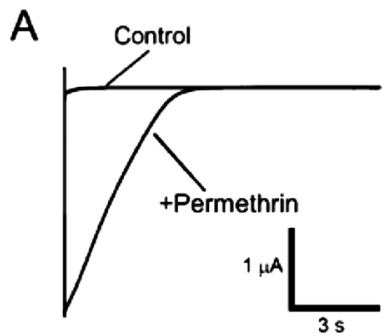

B
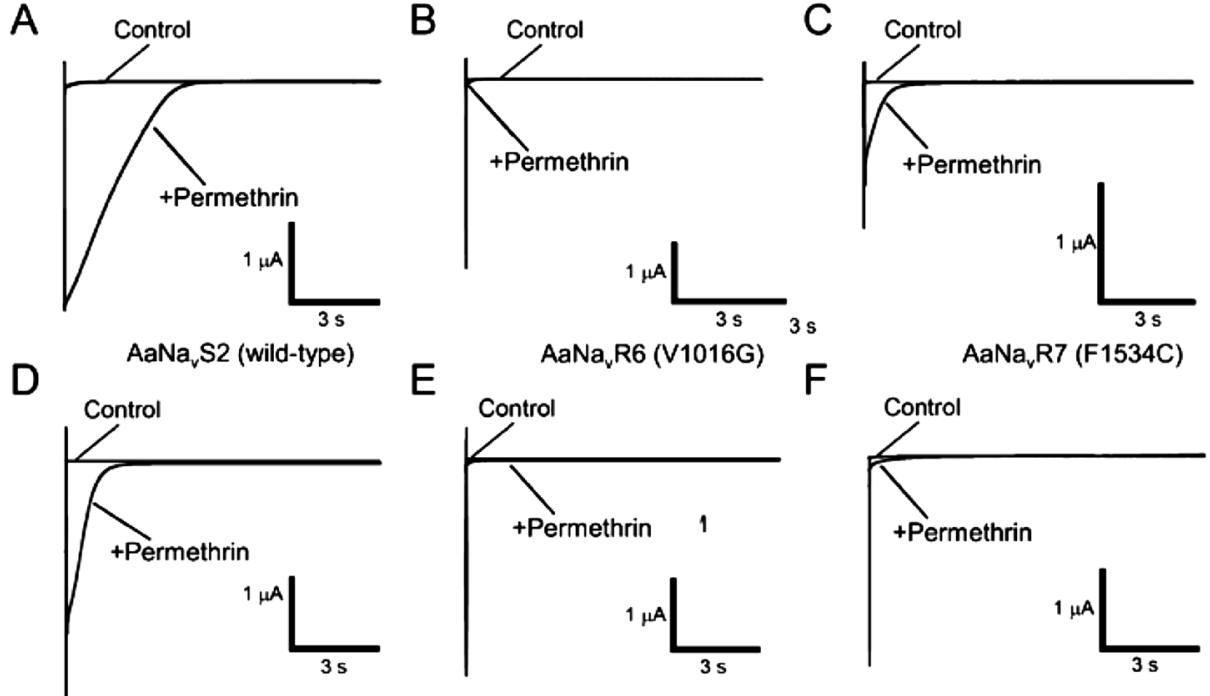

AaNa $\mathrm{R}$ (S989P)

$\mathrm{AaNa}_{\mathrm{v}} \mathrm{R} 9(\mathrm{~S} 989 \mathrm{P}+\mathrm{V} 1016 \mathrm{G})$

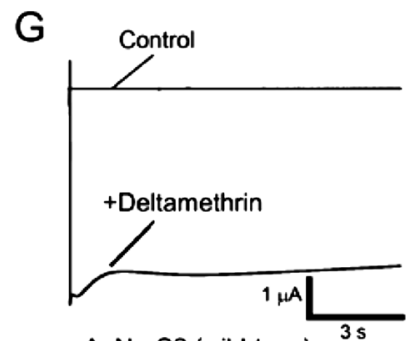

$\mathrm{H}$
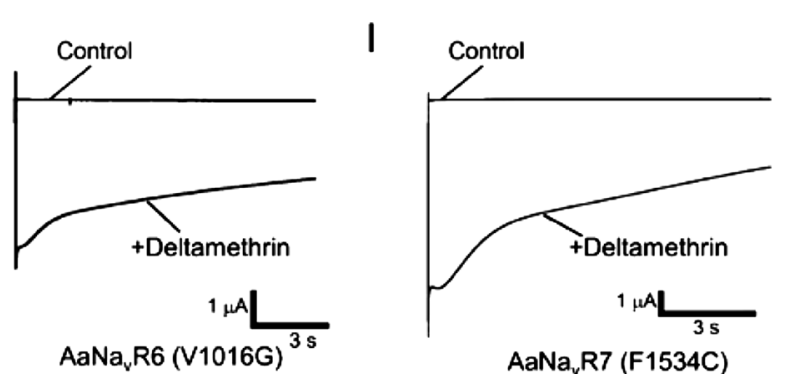

$\mathrm{AaNa}_{\mathrm{v}} \mathrm{S} 2$ (wild-type)

J

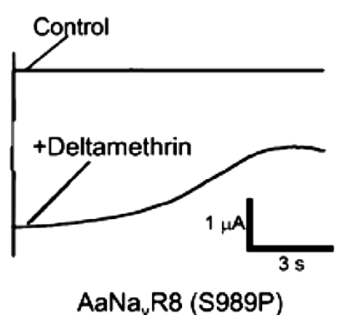

$\mathrm{K}$

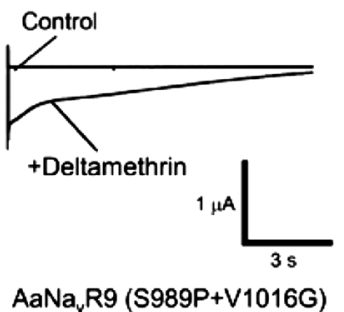

$\mathrm{AaNa}_{\mathrm{v}} \mathrm{R} 7$ (F1534C)

L

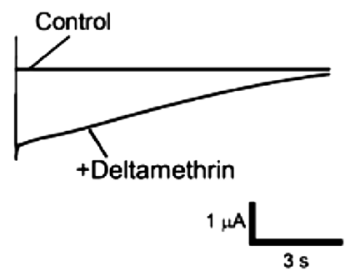

$A a{ }_{2} R 10(S 989 P+V 1016 G+F 1534 C)$

Fig. 2. Pyrethroid-induced tail currents from oocytes injected with various types of voltage-gated sodium channel. (A, G) AaNavS2 (wild type); (B, H) AaNavR6 (V1016G); (C, I) AaNavR7 (F1534C); (D, J), AaNavR8 (S989P); (E, K), AaNavR9 (S989P+V1016G); and (F, L) AaNavR10 $(\mathrm{S} 989 \mathrm{P}+\mathrm{V} 1016 \mathrm{G}+\mathrm{F} 1534 \mathrm{C})$ in the absence (control) or presence of $100 \mathrm{nM}$ permethrin $(\mathrm{A}-\mathrm{F})$ or $100 \mathrm{nM}$ deltamethrin $(\mathrm{G}-\mathrm{L})$.

the high and rapid activity of this class of insecticides in insects, and their low toxicity to mammals. ${ }^{14)}$ However, intensive and frequent use of these chemicals has resulted in the development of resistance to them in A. aegypti. Insecticide resistance in insects is caused by three main mechanisms: (1) reduced sensitivity of the target site, (2) reduced penetration of the insecticide due to altered cuticles, and (3) increased activity or level of detoxification enzymes. Insects develop resistance to insecticides by one or more of these mechanisms. Amino acid substitution in the Vssc, the target site of DDT and pyrethroids, is the best understood mechanism conferring resistance to pyrethroid insecticides. This mechanism is called knockdown resistance $\left.(k d r) .{ }^{15}\right)$ The A. aegypti strain SP, which was discovered in Singapore, was found to exhibit 1650 -fold resistance to permethrin. ${ }^{16)}$ The Vssc in this strain harbors three substitutions (S989P, V1016G,
F1534C, Fig. 1A), but its contribution to pyrethroid resistance is unclear. In this study, we analyzed the pyrethroid sensitivity of Vssc harboring these substitutions, using a TEVC method.

To examine the effects of these three substitutions in Vssc on pyrethroid sensitivity, we constructed five mutants as follows: the three single mutants V1016G (AaNavR6), F1534C (AaNavR7), and S989P (AaNavR8), the double mutant S989P+V1016G (AaNavR9), and the triple mutant S989P+V1016G+F1534C (AaNavR10). ${ }^{17)}$ The effects of the three substitutions (S989P, V1016G, and F1534C), alone or in combination, on the voltage dependence of activation was evaluated. $\mathrm{Na}^{+}$currents recorded in oocytes expressing mutant channels exhibited voltage-dependent activation similar to those expressing the wild type (Table 1).

Pyrethroids are classified as type I or type II according to 
their chemical structure. Type I pyrethroids, such as permethrin, lack an $\alpha$-cyano group at the phenoxybenzyl alcohol position, whereas type II pyrethroids, such as deltamethrin, contain an $\alpha$-cyano-3-phenoxybenzyl alcohol moiety (Fig. 1B). ${ }^{18,19)}$ The decay of tail currents induced by type II pyrethroids (e.g., deltamethrin) is at least one order of magnitude slower than that induced by type I pyrethroids (e.g., permethrin; Fig. 2). Pyrethroid-induced tail currents were elicited with a train of 100 depolarizing pulses. Currents were recorded $10 \mathrm{~min}$ after the application of permethrin (Fig. 2). In all five mutant channels, the same concentration of permethrin induced only a small tail current, which rapidly recovered to the baseline (Fig. 2A-F, Table 1). The V1016G and F1534C substitutions independently reduced the sensitivity of the Vssc to permethrin by 100 - and 25-fold, respectively (Table 1 ). The combination of three substitutions, viz., S989P+V1016G+F1534C, was associated with 1100-fold greater resistance. Deltamethrin-induced tail currents initially increased and then gradually recovered in the wild-type channel (Fig. 2G). However, the current in the S989P+V1016G+F1534C mutant had a lower amplitude and recovered to the baseline more rapidly than did the wild type (Fig. 2L). Compared with the wild-type Vssc, deltamethrin-induced tail currents of four mutant channels (AaNavR6, -R7, -R8, and -R9) decayed very rapidly (Fig. 3H-K). V1016G reduced the sensitivity of the Vssc to deltamethrin by 2-fold, while F1534C alone had no effect on Vssc sensitivity (Table 1). In addition, S989P alone had no effect on deltamethrin sensitivity, but the combination of S989P and V1016G (AaNavR9) reduced the deltamethrin sensitivity of the Vssc by 10-fold, which was a 5-fold reduction in deltamethrin sensitivity compared with that induced by V1016G alone. Furthermore, S989P+V1016G+F1534C (AaNavR10) exhibited 90 -fold greater resistance than the wild type.

In conclusion, the three substitutions S989P, V1016G, and F1534C convey a high level of resistance to pyrethroid. Additionally, these substitutions in the Vssc affected permethrin sensitivity and deltamethrin sensitivity differrently. The combi- nation of these three substitutions may result in an inability to control this key mosquito vector.

\section{2. $\mathrm{nAChR}$ and neonicotinoid resistance of cotton aphid (Aphis gossypii)}

Neonicotinoids have been used for more than 20 years to protect economically important crops. ${ }^{20-22)}$ However, although resistance to neonicotinoids has developed relatively slowly, it is now recognized as an increasingly important issue. ${ }^{23)}$ In most cases, P450-mediated detoxification plays a primary role in insecticide resistance in a variety of insects, including the silverleaf whitefly (Bemisia tabaci) and the Colorado potato beetle (Leptinotarsa decemlineata). ${ }^{24,25)}$ On the other hand, laboratory selection of brown planthopper (Nilaparvata lugens) using imidacloprid resulted in the identification of an $\alpha$-subunit Y151S mutation that was responsible for the acquisition of neonicotinoid resistance. ${ }^{26)}$ However, this mutation has not been identified in any pest in the field. Instead, a mutation in the $\mathrm{nAChR} \beta 1$ subunit was found to be a major factor in imidacloprid resistance in a field population of peach-potato aphids (Myzus persicae). ${ }^{27)}$ The emergence of neonicotinoid resistance was recently reported in an A. gossypii (Kushima) clone in Miyazaki Prefecture, Japan. ${ }^{28)}$ However, the mechanisms of neonicotinoid resistance have remained unknown, so in this study, we attempted to identify them.

The A. gossypii (Kushima) clone exhibited resistance to neonicotinoid insecticides compared with a susceptible clone (Table 2). ${ }^{29)}$ Interestingly, the Kushima clone had a high level of resistance to nitro-substituted neonicotinoids (Fig. 3A), such as imidacloprid. In contrast, the Kushima clone exhibited low to moderate levels of resistance to cyano-substituted neonicotinoids (e.g., acetamiprid). The $\mathrm{LC}_{50}$ values of acetamiprid and imidacloprid for the Kushima clone without piperonyl butoxide (PBO) pretreatment were 6.54 and $75.7 \mathrm{ppm}$, but 1.91 and $7.01 \mathrm{ppm}$ with $\mathrm{PBO}$, respectively. The synergistic factor of acetamiprid was lower than that for imidacloprid (3.4 vs. 11, respectively). These

Table 2. Insecticidal activity of neonicotinoids to susceptible aphids and the Kushima clone with and without pretreatment with piperonyl butoxide $(\mathrm{PBO})$

\begin{tabular}{|c|c|c|c|c|c|c|}
\hline \multirow{2}{*}{ Compound } & \multicolumn{2}{|r|}{ Susceptible clone } & \multicolumn{2}{|r|}{ Kushima clone } & \multirow{2}{*}{$\mathrm{RF}^{\mathrm{a})}$} & \multirow{2}{*}{$\mathrm{SF}^{\mathrm{b})}$} \\
\hline & $\mathrm{LC}_{50}(\mathrm{ppm})$ & $95 \%$ confidence limit of $\mathrm{LC}_{50}$ & $\mathrm{LC}_{50}(\mathrm{ppm})$ & $95 \%$ confidence limit of $\mathrm{LC}_{50}$ & & \\
\hline Acetamiprid & 0.10 & $0.080-0.12$ & 6.54 & $4.62-9.73$ & 65.4 & \\
\hline Thiacloprid & 0.30 & $0.20-0.61$ & 7.15 & $4.40-11.8$ & 23.8 & \\
\hline Imidacloprid & 0.35 & $0.20-0.85$ & 75.7 & $44.7-193$ & 216 & \\
\hline Clothianidin & 0.10 & $0.061-0.18$ & 39.4 & $28.0-58.5$ & 394 & \\
\hline Thiamethoxam & 0.19 & $0.12-0.31$ & 56.0 & $40.6-73.7$ & 295 & \\
\hline Dinotefuran & 0.66 & $0.40-1.04$ & 167 & $114-285$ & 253 & \\
\hline Nitenpyram & 0.30 & $0.19-0.54$ & 71.0 & $27.5-103$ & 237 & \\
\hline Acetamiprid+PBO & 0.028 & $0.016-0.048$ & 1.91 & $1.03-3.08$ & & 3.4 \\
\hline Imidacloprid+PBO & 0.050 & $0.032-0.079$ & 7.01 & $3.46-12.0$ & & 11 \\
\hline
\end{tabular}

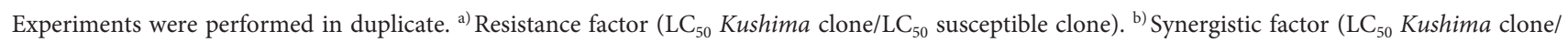
$\mathrm{LC}_{50}$ Kushima clone with PBO). 
A

\section{Cyano-substituted neonicotinoid}<smiles>CC(=NC#N)N(C)Cc1ccc(Cl)nc1</smiles>

Acetamiprid<smiles>N#CN=C1SCCN1Cc1ccc(Cl)nc1</smiles>

Thiacloprid

Nitro-substituted

neonicotinoid<smiles></smiles>

Imidacloprid<smiles>CN/C(=N\[N+](=O)[O-])NCc1cnc(Cl)s1</smiles>

Clothianidin<smiles></smiles><smiles>CNC(=N[N+](=O)[O-])NCC1CCOC1</smiles>

Dinotefuran<smiles>CCN(Cc1ccc(Cl)nc1)/C(=C/[N+](=O)[O-])NC</smiles>

Nitenpyram

B

Thiamethoxam

\begin{tabular}{|c|c|c|c|c|c|c|c|c|c|c|c|}
\hline \multirow[b]{2}{*}{ Species } & \multicolumn{11}{|c|}{ Amino acid number of Gallus gallus $\beta 2$ subunit } \\
\hline & 73 & 74 & 75 & 76 & 77 & 78 & 79 & 80 & 81 & 82 & 83 \\
\hline Homo sapiens $\beta 2$ & $\mathrm{~N}$ & V & W & $\mathrm{L}$ & $T$ & Q & $\mathrm{E}$ & W & $E$ & $\mathrm{D}$ & $\mathrm{Y}$ \\
\hline Gallus gallus $\beta 2$ & $\mathrm{~N}$ & V & W & L & $\mathbf{T}$ & Q & $E$ & W & $E$ & D & Y \\
\hline \multirow[t]{3}{*}{ Rattus norvegicus $\beta 2$} & $\mathrm{~N}$ & $\mathrm{~V}$ & $\mathrm{~W}$ & $\mathrm{~L}$ & $\mathbf{T}$ & $\mathrm{Q}$ & $\mathrm{E}$ & $W$ & $E$ & $\mathrm{D}$ & Y \\
\hline & \multicolumn{5}{|c|}{ Amino acid number of } & \multicolumn{6}{|c|}{ Aphis gossypii $\beta 1$ subunit } \\
\hline & 77 & 78 & 79 & 80 & 81 & 82 & 83 & 84 & 85 & 86 & 87 \\
\hline Drosophila melanogaster $\beta 1$ & C & V & W & $\mathrm{L}$ & $\mathbf{R}$ & L & V & W & Y & D & $\mathrm{Y}$ \\
\hline Bemisia tabaci $\beta 1$ & $\mathrm{~N}$ & V & W & L & $\mathbf{R}$ & L & V & W & $\mathrm{N}$ & $\mathrm{D}$ & Y \\
\hline Myzus persicae $\beta 1$ & $\mathrm{~N}$ & V & W & L & $\mathbf{R}$ & L & V & W & $\mathrm{R}$ & D & Y \\
\hline Aphis gossypii $\beta 1$ & $\mathrm{~N}$ & V & W & L & $\mathbf{R}$ & L & V & W & $\mathrm{R}$ & $\mathrm{D}$ & Y \\
\hline Aphis gossypii Kushima $\beta 1$ & $\mathrm{~N}$ & $\mathrm{~V}$ & W & $\mathrm{L}$ & $T$ & $\mathrm{~L}$ & $\mathrm{~V}$ & W & $\mathrm{R}$ & D & Y \\
\hline
\end{tabular}

Fig. 3. Chemical structures of neonicotinoid insecticides and alignment of amino acid sequences in loop D of the agonist binding region of vertebrate and insect nicotinic ACh receptor subunits (nAChRs). Homo sapiens (human), Gallus gallus (chicken), Rattus norvegicus (rat), Drosophila melanogaster (fruit fly), Bemisia tabaci (sweet potato whitefly), Myzus persicae (peach-potato aphid), and Aphis gossypii (cotton aphid).

Table 3. $\mathrm{pEC}_{50}$ and $I_{\max }$ values of ACh, acetamiprid, and imidacloprid for wild-type, T77R, and T77R+E79V D $\alpha 2 \beta 2 \mathrm{nAChRs}$

\begin{tabular}{|c|c|c|c|c|c|c|}
\hline \multirow{3}{*}{$\begin{array}{l}\text { Compounds } \\
\text { Ach }\end{array}$} & \multicolumn{2}{|c|}{$\mathrm{D} \alpha 2 \beta 2 \mathrm{WT}$} & \multirow{2}{*}{\multicolumn{2}{|c|}{$\mathrm{D} \alpha 2 \beta 2 \mathrm{~T} 77 \mathrm{R}$}} & \multirow{2}{*}{\multicolumn{2}{|c|}{$\mathrm{D} \alpha 2 \beta 2 \mathrm{~T} 77 \mathrm{R}+\mathrm{E} 79 \mathrm{~V}$}} \\
\hline & \multirow{2}{*}{$\frac{\mathrm{pEC}_{50}}{4.60 \pm 0.04}$} & \multirow{2}{*}{$\frac{I_{\max }}{1.08 \pm 0.03}$} & & & & \\
\hline & & & $4.64 \pm 0.07$ & $1.03 \pm 0.01$ & $4.47 \pm 0.03$ & $1.04 \pm 0.02$ \\
\hline Acetamiprid & $5.26 \pm 0.09$ & $0.49 \pm 0.03$ & $5.42 \pm 0.06$ & $0.99 \pm 0.04 *$ & $5.38 \pm 0.04$ & $0.97 \pm 0.03 *$ \\
\hline Imidacloprid & $5.61 \pm 0.06$ & $0.77 \pm 0.03$ & $6.35 \pm 0.06 *$ & $0.79 \pm 0.03$ & $6.18 \pm 0.02 *$ & $0.96 \pm 0.02 *$ \\
\hline
\end{tabular}

Values shown are the results of a fit of the concentration-response data (mean \pm S.E.M., $n=4$ ). Statistical test (one-way ANOVA, Dunnett's multiplecomparison test) is for significant differences from the wild-type data. ${ }^{*} p<0.05$. The peak amplitude of the current recorded in the response to each challenge was normalized to the maximum amplitude of the response to ACh. Data from the D $\alpha 2 \beta 2$, T77R, and T77R+E79V mutants were normalized to the response to $1 \mathrm{mM}$ ACh. 
A

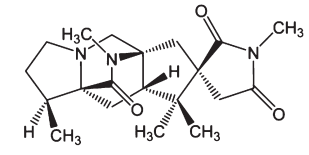

B

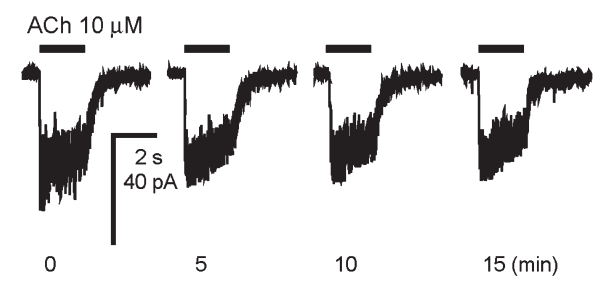

D

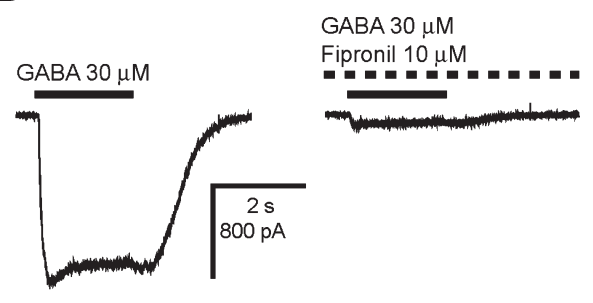

F

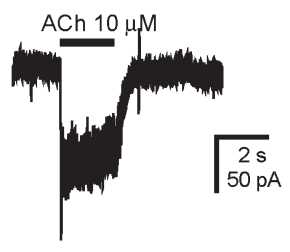

H
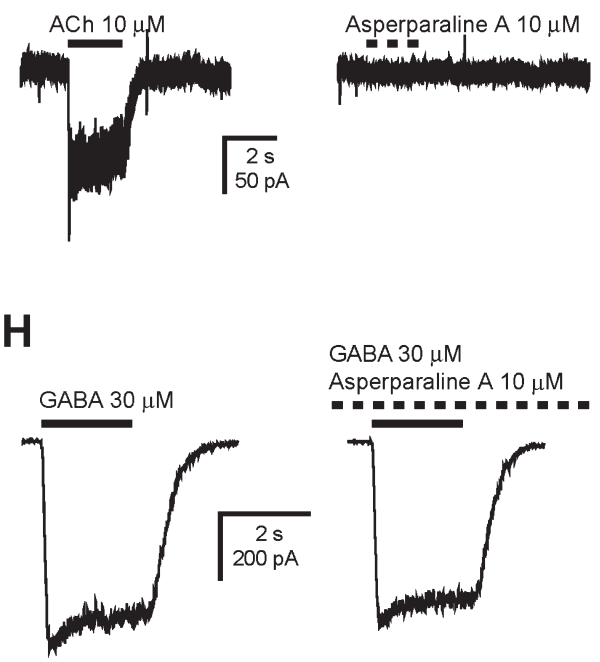

GABA $30 \mu \mathrm{M}$
Asperparaline A $10 \mu \mathrm{M}$

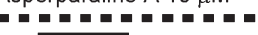

C

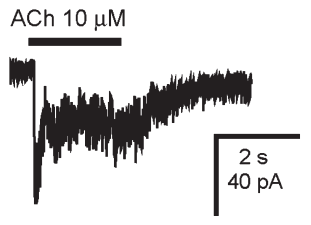

E

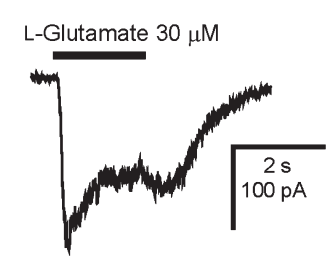

L-Glutamate $30 \mu \mathrm{M}$

Fipronil $10 \mu \mathrm{M}$
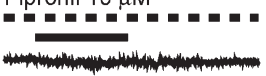

G

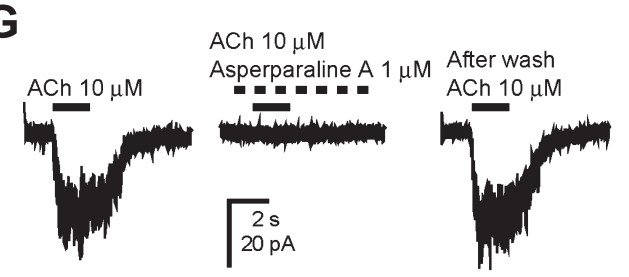

I
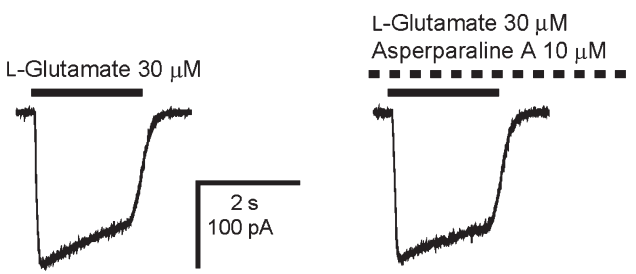

Fig. 4. Chemical structure of asperparaline $A$ and acetylcholine (ACh)-induced currents. (A) Chemical structure of asperparaline A. (B) Acetylcholine (ACh)-induced currents. The effects of blockers (mecamylamine and fipronil) on the ACh-(C), $\gamma$-aminobutyric acid (GABA)-(D), and L-glutamate-induced currents (E) and the actions of asperparaline A on the resting-state (F) and neurotransmitter-evoked currents (G-I) in silkworm (Bombyx mori) larval neurons.

results indicate that the Kushima clone acquired a mechanism of resistance other than enhanced oxidative metabolism.

The N-terminal regions of the Kushima clone nAChR subunits, which encompass the conserved domains (loop A-F) that comprise the ACh and neonicotinoid binding sites, were sequenced. Although a limited number of silent single-nucleotide polymorphisms were detected, no amino acid changes were observed. In contrast, a single point mutation, Arg81 (AGA) to Thr
(ACA), was detected in the $\beta 1$ subunit (Fig. 3B).

To examine the effects of this R81T mutation in the $\beta 1$ subunit on neonicotinoid agonist activity, we used the $\mathrm{D} \alpha 2$-vertebrate $\beta 2$ hybrid receptor for the following reasons: Insect $\mathrm{nAChR}$ functional expression is difficult, but the fruit fly (Drosophila melanogaster) D $\alpha 2$-chicken (Gallus gallus) $\beta 2$ hybrid receptor has been well established for assaying neonicotinoids. We constructed an equivalent single mutant (T77R) of the 
chicken $\beta 2$ subunit (Fig. 3B) and also evaluated the T77R+E79V double mutant $(\mathrm{T} 77 \mathrm{R}+\mathrm{E} 79 \mathrm{~V})$ because the cotton aphid $\mathrm{nAChR}$ $\beta 1$ subunit has $\mathrm{Val}$ (Val83) at the equivalent amino acid residue of the chicken $\beta 2$ subunit (E79). The E79 residue of the chicken $\beta 2$ subunit may suppress interactions between the nitro group of neonicotinoids and Arg 77 through the negative electrostatic forces of Glu79. ${ }^{10)}$ The T77R and T77R+E79V mutations did not significantly affect the $\mathrm{pEC}_{50}$ and $I_{\max }$ values for the natural agonist ACh (Table 3), compared with those of the wild-type $\mathrm{D} \alpha 2 \beta 2 \mathrm{nAChR}$ (WT). The $\mathrm{pEC}_{50}$ values of acetamiprid and imidacloprid for wild-type $\mathrm{D} \alpha 2 \beta 2 \mathrm{nAChR}$ were $5.26 \pm 0.09$ and $5.61 \pm 0.06$, respectively. On the other hand, the equivalent values for the $\mathrm{D} \alpha 2 \beta 2 \mathrm{nAChR}$ single mutant (T77R) were 5.42 \pm 0.06 and 6.35 \pm 0.06 , and $5.38 \pm 0.04$ and $6.18 \pm 0.02$ for the $\mathrm{D} \alpha 2 \beta 2$ $\mathrm{nAChR}$ double mutant $(\mathrm{T} 77 \mathrm{R}+\mathrm{E} 79 \mathrm{~V})$, respectively. The $\mathrm{pEC}_{50}$ value of imidacloprid was significantly shifted relative to that of the wild type, but that for acetamiprid exhibited no significant change (Table 3 ).

In conclusion, the mutation of Arg81 (AGA) to Thr (ACA) conveys a high level of resistance to neonicotinoids. This mutation, which had no effect on the $\mathrm{pEC}_{50}$ values of ACh, may provide both a high level of resistance to neonicotinoids and a low fitness cost. Additionally, the differences in the resistance factors (RFs) between nitro- and cyano-substituted neonicotinoids were likely due to the effect of the R81T mutation on agonist affinity. The different effects of imidacloprid (nitro-substituted) and acetamiprid (cyano-substituted) are probably due to their slightly different interactions with nAChRs.

\section{Mode of action of fungal metabolite asperparaline $\mathrm{A}$}

Asperparalines are alkaloids produced by Aspergillus japonicus JV-23 when grown on 'okara' medium (soybean residue resulting from tofu manufacturing). They are known to paralyze silkworm (Bombyx mori) larvae when administered orally using artificial diets. ${ }^{30}$ The unique structures of asperparalines have been characterized (Fig. 4A), but their targets have not yet been elucidated. In this study, we thus attempted to identify the mode of action of asperparaline A.

It is assumed that the target of asperparaline $\mathrm{A}$ is in the nervous system or neuromuscular junction, since this compound induces paralysis in silkworm larvae. By applying whole-cell patch-clamp electrophysiology to larval neurons of $B$. mori, we were able to record the neurotransmitter-evoked responses of native ligand-gated ion channels and study the actions of asperparaline A. ${ }^{31)}$ Application of ACh $(10 \mu \mathrm{M})$ resulted in a rapid inward current with fast and slow desensitizing phases. The AChinduced currents were stably recorded for $15 \mathrm{~min}$ or longer (Fig. $4 \mathrm{~B})$. The entire current was completely blocked by bath-applied $100 \mu \mathrm{M}$ mecamylamine (Fig. 4C), a non-competitive antagonist of nAChRs. Both $\gamma$-aminobutyric acid (GABA)- and L-glutamate-induced currents were attenuated by bath-applied fipronil, a phenylpyrazole insecticide known to block the chloride channels of GABA- and L-glutamate-gated chloride channels in insects (Fig. 4D, E). To examine whether asperparaline A activates any of the ligand-gated ion channels expressed in silkworm neurons, it was applied alone to these neurons at $10 \mu \mathrm{M}$. The compound had no effect on the membrane current amplitude to clamp the membrane potential of the $B$. mori larval neurons (Fig. 4F). Hence, it was also bath-applied for $1 \mathrm{~min}$, prior to coapplication for $2 \mathrm{sec}$ with ACh $(10 \mu \mathrm{M})$, L-glutamate $(30 \mu \mathrm{M})$, and $\mathrm{GABA}(30 \mu \mathrm{M})$, to explore any possible antagonist activity on ligand-gated ion channels present in the neurons. Asperparaline A markedly and reversibly blocked the ACh-induced current when applied at $1 \mu \mathrm{M}$ (Fig. $4 \mathrm{G}, \mathrm{IC}_{50}=20.2 \mathrm{nM}$ ). However, it barely affected the peak current amplitude of GABA (Fig. 4H) and the L-glutamate-evoked responses (Fig. 4I) were barely affected by $10 \mu \mathrm{M}$ bath-applied asperparaline $\mathrm{A}$.

To explore further the blocking action, the concentration-response relationship of $\mathrm{ACh}$ was measured in the absence and presence of $100 \mathrm{nM}$ asperparaline A (Fig. 5). Asperparaline A reduced the normalized maximum response to ACh to approximately $22.2 \%$, while scarcely influencing pEC $_{50}$ (with $100 \mathrm{nM}$ asperparaline A, 4.98 $\pm 0.14, \mathrm{EC}_{50}=10.5 \mu \mathrm{M}$; without asperparaline $\left.\mathrm{A}, 4.94 \pm 0.04, \mathrm{EC}_{50}=11.4 \mu \mathrm{M}\right)$. No significant shift in $\mathrm{pEC}_{50}$ was observed in association with the presence of $100 \mathrm{nM}$ asperparaline A. Additionally, asperparaline A was tested on chicken $\alpha 3 \beta 4, \alpha 4 \beta 2$, and $\alpha 7 \mathrm{nAChRs}$ expressed in Xenopus laevis oocytes. Asperparaline A was inactive or only weakly active on these three nAChRs.

In conclusion, asperparaline A from A. japonicas JV-23 acts on insect $\mathrm{nAChRs}$ as a non-competitive antagonist, and is highly selective to insect nAChRs over vertebrate ones. Additionally, it is probably active on the insects R $81 \mathrm{~T}$ mutation.

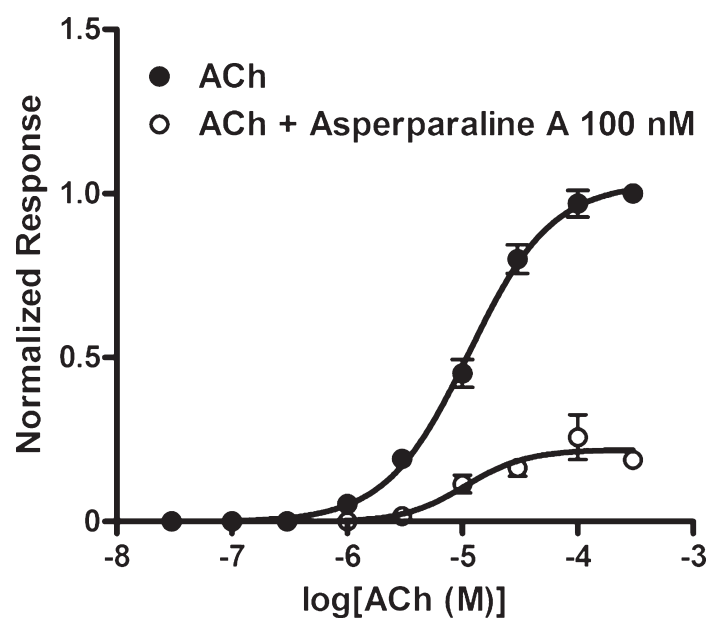

Fig. 5. Effects of asperparaline A on the concentration-response curve for ACh in silkworm (Bombyx mori) larval neurons. The ACh-induced responses were measured at various concentrations in the presence and absence of $100 \mathrm{nM}$ asperparaline $\mathrm{A}$. The $\mathrm{pEC}_{50}\left[=\log \left(1 / \mathrm{EC}_{50}\right)\right]$ values determined in the presence and absence of asperparaline A were $4.98 \pm 0.10$ $\left(n=4, \mathrm{EC}_{50}=10.5 \mu \mathrm{M}\right)$ and $4.94 \pm 0.04\left(n=7, \mathrm{EC}_{50}=11.4 \mu \mathrm{M}\right)$, respectively. No significant shift in $\mathrm{EC}_{50}$ was observed in association with the application of asperparaline A. 


\section{Conclusion}

In the present study, the mechanism of resistance to pyrethroids in A. aegypti, that to neonicotinoids in A. gossypii, and the mechanism of action of the natural product asperparaline A were clarified. We also demonstrated that similar compounds that interacted on the same target with slightly different substituents exhibited different effects on the target molecule with different amino acid residues at the activity site, and that natural products exhibited specific activity and selectivity to insect ion channels.

We plan to undertake further research on novel insecticides, mainly focusing on the mechanisms of action in order to contribute to pest control and improvement in agricultural production.

\section{Acknowledgements}

This study was carried out at the Faculty of Agriculture, Kindai University, and Odawara Research Center, Nippon-soda Co., Ltd. The author would like to express his gratitude to Kazuhiko Matsuda for his kind guidance and warm encouragement during the course of this study. The author is also greatly indebted to Takashi Tomita, Shinji Kasai, Osamu Komagata, Kentaro Itokawa (National Institute of Infectious Diseases), Akira Matsuura (Miyazaki Agricultural Research Institute), Satoshi Toda (NARO Institute of Fruit Tree Science), and Hideo Hayashi (Osaka Prefecture University) for their kind suggestions and support for this research. Finally, the author would like to offer special thanks to the members of Nipponsoda Co., Ltd., who created a beneficial work environment and supported this research. The work on $\mathrm{nAChR}$ and the neonicotinoid resistance of Aphis gossypii was supported by a grant from the Ministry of Agriculture, Forestry, and Fisheries of Japan (Genomics-based Technology for Agricultural Improvement, PRM-003).

\section{References}

1) G. Feng, P. Deak, M. Chopra and L. M. Hall: Cell 82, 1001-1011 (1995).

2) E. Zlotkin: Annu. Rev. Entomol. 44, 429-455 (1999).

3) T. Narahashi: Pharmacol. Toxicol. 79, 1-14 (1996).

4) A. Karlin: Nat. Rev. Neurosci. 3, 102-114 (2002).

5) M. Tomizawa and J. E. Casida: Pest Manag. Sci. 57, 914-922 (2001).

6) K. Matsuda, S. D. Buckingham, J. C. Freeman, M. D. Squire, H. A. Baylis and D. B. Sattelle: Br. J. Pharmacol. 123, 518-524 (1998).

7) K. Matsuda, S. D. Buckingham, D. Kleier, J. J. Rauh, M. Grauso and D. B. Sattelleemail: Trends Pharmacol. Sci. 22, 573-580 (2001).
8) M. Tomizawa and J. E. Casida: Annu. Rev. Pharmacol. Toxicol. 45, 247-268 (2005).

9) K. Matsuda, M. Shimomura, M. Ihara, M. Akamatsu and D. B. Sattelle: Biosci. Biotechnol. Biochem. 69, 1442-1452 (2005).

10) M. Shimomura, M. Yokota, M. Ihara, M. Akamatsu, D. B. Sattelle and K. Matsuda: Mol. Pharmacol. 70, 1255-1263 (2006).

11) J. G. Scott: Insect Biochem. Mol. Biol. 29, 757-777 (1999).

12) J. E. Casida and K. A. Durkin: Annu. Rev. Entomol. 58, 99-117 (2013).

13) C. Bass, A. M. Puinean, C. T. Zimmer, I. Denholm, L. M. Field, S. P. Foster, O. Gutbrod, R. Nauen, R. Slater and M. S. Williamson: Mol. Biol. 51, 41-51 (2014).

14) J. S. Mackenzie, D. J. Gubler and L. R. Petersen: Nat. Med. 10(Suppl), S98-S109 (2004).

15) M. S. Williamson, I. Denholm, C. A. Bell and A. L. Devonshire: Mol. Gen. Genet. 240, 17-22 (1993).

16) S. Kasai, O. Komagata, K. Itokawa, T. Shono, M. Kobayashi, T. Tomita and L. C. Ng: PLoS Negl. Trop. Dis. 8, e2948 (2014).

17) K. Hirata, O. Komagata, K. Itokawa, T. Tomita, S. Kasai and A. Yamamoto: PLoS Negl. Trop. Dis. 8, e3085 (2014).

18) Z. Hu, Y. Du, Y. Nomura and K. Dong: Insect Biochem. Mol. Biol. 41, 9-13 (2011).

19) T. Narahashi: J. Pharmacol. Exp. Ther. 294, 1-26 (2000).

20) P. Jeschke and R. Nauen: Pest Manag. Sci. 64, 1084-1098 (2008).

21) R. Nauen, I. Denholm, T. Dennehy and R. Nichols: Pest Manag. Sci. 64, 1082-1083 (2008).

22) A. Elbert, M. Haas, B. Springer, W. Thielert and R. Nauen: Pest Manag. Sci. 64, 1099-1105 (2008).

23) R. Nauen and I. Denholm: Arch. Insect Biochem. Physiol. 58, 200-215 (2005).

24) N. Rauch and R. Nauen: Arch. Insect Biochem. Physiol. 54, 165-176 (2003).

25) J.-Z. Zhao, B. A. Bishop and E. J. Grafius: J. Econ. Entomol. 93, 15081514 (2000).

26) Z. Liu, M. S. Willamson, S. J. Lansdell, I. Denholm, Z. Han and N. S. Millar: Proc. Natl. Acad. Sci. U.S.A. 102, 8420-8425 (2005).

27) C. Bass, A. M. Puinean, M. Andrews, P. Cutler, M. Daniels, J. Elias, V. L. Paul, A. J. Crossthwaite, I. Denholm, L. M. Field, S. P. Foster, R. Lind, M. S. Williamson and R. Slater: BMC Neurosci. 12, 51-62 (2011).

28) A. Matsuura and M. Nakamura: Appl. Entomol. Zool. (Jpn.) 49, 535540 (2014).

29) K. Hirata, R. Kiyota, A. Matsuura, S. Toda, A. Yamamoto and T. Iwasa: J. Pestic. Sci. 40, 25-31 (2015).

30) H. Hayashi, Y. Nishimoto and H. Nozaki: Tetrahedron Lett. 38, 56555658 (1997).

31) K. Hirata, S. Kataoka, S. Furutani, H. Hayashi and K. Matsuda: PLoS ONE 6, e18354 (2011). 